M. Droste, J. K. Truss

\title{
THE UNCOUNTABLE COFINALITY OF THE AUTOMORPHISM GROUP OF THE COUNTABLE UNIVERSAL DISTRIBUTIVE LATTICE
}

Dedicated to Rüdiger Göbel on the occasion of his 70th birthday.

\begin{abstract}
We show that the automorphism group of the countable universal distributive lattice has strong uncountable cofinality, and we adapt the method to deduce the strong uncountable cofinality of the automorphism group of the countable universal generalized boolean algebra.
\end{abstract}

\section{Introduction}

In [6] a detailed analysis was given of the automorphism group of the countable universal homogeneous distributive lattice $\mathbb{D}$. In particular, its normal subgroups were determined, and the small index property was verified. The question of whether Aut $(\mathbb{D})$ has uncountable cofinality was however left open, and it is our purpose in this paper to establish this. The same methods are used to demonstrate the uncountable cofinality of the automorphism group of the countable universal homogeneous generalized boolean algebra.

The background to this problem is explained in [7], but we recap here on the main points. A group $G$ is said to have uncountable cofinality if it cannot be written as the union of a countable chain of proper subgroups. This notion has been studied by a number of authors, originally Koppelberg and Tits [13], who, in response to a question of Serre, proved the uncountable cofinality of the direct power of infinitely many copies of a finite perfect group. Macpherson and Neumann [14] established the same result for the

2010 Mathematics Subject Classification: 06A07, 06F15, 20B27, $20 \mathrm{E} 15$.

Key words and phrases: cofinality, lattice, boolean algebra.

This work was supported by EPSRC grant EP/H00677X/1. 
symmetric group on a countably infinite set, and building on these methods, uncountable cofinality has been established in a number of other cases, for instance the automorphism group $A(\mathbb{Q})$ of the rational numbers as an ordered set [10], for other (partially or totally) ordered sets [5, 7], and for the homeomorphism groups of certain topological spaces [4]. For a survey of this field, see Thomas [15]. A rather stronger condition is that $G$ have strong uncountable cofinality, and this means that $G$ cannot be written as the union of an ascending chain of proper subsets $\left(U_{n}: n \in \omega\right)$ each closed under formation of inverses, and such that for each $i$ and $j$, there is $k$ such that $U_{i} U_{j} \subseteq U_{k}$. It was shown in [5] that strong uncountable cofinality is equivalent to uncountable cofinality together with a property introduced in [2] called 'Bergman's property': for any generating set $E$ for $G$ which contains the identity and is closed under inverses, there is $n \in \mathbb{N}$ such that $G=E^{n}$. This property has also found considerable recent interest, cf., e.g., $[2,4,5,12]$. We work here exclusively with strong uncountable cofinality, thereby establishing for the present automorphism groups both uncountable cofinality and the Bergman property.

\section{The uncountable cofinality of the automorphism group of the countable universal homogeneous distributive lattice}

In this section we shall establish the uncountable cofinality of Aut $(\mathbb{D})$. This follows a combination of methods used in other cases, principally those of the rationals [10] and the countable atomless boolean algebra [4]. For these we require the following definitions.

First we recall what $\mathbb{D}$ is. It is known that the class of finite distributive lattices is an amalgamation class, so it follows by the general Fraïssé theory (see [11] for instance) that there is a unique countable universal homogeneous distributive lattice, which we denote by $\mathbb{D}$. This has no greatest or least element, all the maximal chains are isomorphic to $\mathbb{Q}$, and any 'interval' $[a, b]=\{x \in \mathbb{D}: a \leq x \leq b\}$ is itself a lattice, which is isomorphic to the countable atomless boolean algebra. Modifying this a little, we may also consider the class of finite 'generalized boolean algebras', which are finite distributive lattices with a least element 0 . This is an amalgamation class under the class of maps which are required to fix 0 (as well as the lattice operations $\wedge$ and $\vee$ ), and the resulting structure is a universal homogeneous generalized boolean algebra $\mathbb{B}$. For this structure, there is a least but no greatest element, all maximal chains are isomorphic to the rational interval $[0,1)$, and again any interval is isomorphic to the countable atomless boolean algebra. Both $\mathbb{D}$ and $\mathbb{B}$ are 'relatively complemented', which means that for any $a \leq x \leq b$ there is $y \in[a, b]$ such that $x \wedge y=a$ and $x \vee y=b$. We shall quote various results proved in [6] about $\mathbb{D}$ and $\mathbb{B}$. 
By a coterminal $\mathbb{Z}$-chain in $\mathbb{D}$ (or more generally in any partially ordered set) we understand a family $\left\{a_{i}: i \in \mathbb{Z}\right\}$ indexed by the integers, such that $a_{i}<a_{i+1}$ for each $i$, and for every $x \in \mathbb{D}$, there are $i$ and $j$ such that $a_{i} \leq x \leq a_{j}$. A moiety is a subset of $\mathbb{D}$ of the form $\bigcup_{n \in \mathbb{Z}}\left(a_{2 n}, a_{2 n+1}\right)$, for some coterminal $\mathbb{Z}$-chain $\left\{a_{i}: i \in \mathbb{Z}\right\}$. The sets $\left(a_{2 n}, a_{2 n+1}\right)$ are called 'components' of the moiety. The notion of moiety was originally introduced by Neumann [3] in the proof of the small index property for the symmetric group on $\omega$, signifying a set which is 'half' of the whole, and versions of the same idea have appeared in many other similar proofs of the small index property or uncountable cofinality.

For any permutation group $G$, we denote the setwise and pointwise stabilizers of a subset $A$ of the set on which $G$ acts by $G_{\{A\}}$ and $G_{A}$ respectively.

We need the following result, which we give without proof. This is similar to Lemmas 2.2 and 2.3 in [6], adapted for our current purposes.

Lemma 2.1. For $i=1,2$, let $L_{i}$ be a relatively complemented distributive lattice, and $L_{i}^{\prime} \subseteq L_{i}$ a sublattice such that the smallest relatively complemented sublattice of $L_{i}$ containing $L_{i}^{\prime}$ is $L_{i}$ itself. Then each isomorphism from $L_{1}^{\prime}$ to $L_{2}^{\prime}$ extends uniquely to an isomorphism of $L_{1}$ to $L_{2}$.

THEOREM 2.2. The automorphism group of the countable universal homogeneous distributive lattice $\mathbb{D}$ has strong uncountable cofinality.

Proof. Let $U_{0} \subseteq U_{1} \subseteq U_{2} \subseteq \ldots \subseteq \operatorname{Aut}(\mathbb{D})=G$ have union equal to $G$ and be such that $U_{n}^{-1}=U_{n}$ and $(\forall i, j)(\exists k) U_{i} U_{j} \subseteq U_{k}$. We aim to show that $U_{n}=G$ for some $n$. As in [7], this is accomplished in a series of steps. By [9] Thm.II.4.20, the countable atomless boolean algebra $\mathbb{B}$ is generated by some maximal chain. Now choose a coterminal $\mathbb{Z}$-chain $\left(a_{n}\right)_{n \in \mathbb{Z}}$ in $\mathbb{D}$. Then each $\left[a_{n}, a_{n+1}\right]$ is isomorphic to $\mathbb{B}$, so we let $C_{n}$ be a maximal chain of $\left[a_{n}, a_{n+1}\right]$ which generates $\left[a_{n}, a_{n+1}\right]$ as a boolean algebra. We remark that $C=\bigcup_{n \in \mathbb{Z}} C_{n}$ generates $\mathbb{D}$ as a relatively complemented lattice, as follows by the argument given in the proof of Lemma 2.3 in [6], and we fix this $C$ in what follows.

(1) Any isomorphism $f$ from $C$ to $C$ extends uniquely to an automorphism of $\mathbb{D}$.

This is immediate by Lemma 2.1 from the fact that $C$ generates $\mathbb{D}$ as a relatively complemented lattice.

(2) Let $\left(a_{n}\right),\left(a_{n}^{\prime}\right)$ be coterminal $\mathbb{Z}$-chains in $C$. Then any isomorphism $f$ from $C \cup \bigcup_{n \in \mathbb{Z}}\left[a_{2 n}, a_{2 n+1}\right]$ to $C \cup \bigcup_{n \in \mathbb{Z}}\left[a_{2 n}^{\prime}, a_{2 n+1}^{\prime}\right]$ extends uniquely to an automorphism of $\mathbb{D}$.

This is proved by the same method as (1).

(3) There is $m_{0}$ such that $G_{\{C\}} \subseteq U_{m_{0}}$. 
To prove this we consider the intersections of the sequence $\left(U_{n}\right)$ with the setwise stabilizer $G_{\{C\}}$ of $C$ in $G$. Since $C \cong \mathbb{Q}$, and by $(1), G_{\{C\}} \cong A(\mathbb{Q})$. By the strong uncountable cofinality of $A(\mathbb{Q})[5]$ we deduce that there is $m_{0}$ such that $U_{m_{0}} \cap G_{\{C\}}=G_{\{C\}}$, which gives the result.

(4) There are a moiety $M=\bigcup_{n \in \mathbb{Z}}\left(a_{2 n}, a_{2 n+1}\right)$ such that each $a_{n}$ lies in $C$, and an integer $m_{1} \geq m_{0}$, such that every automorphism fixing $C \backslash M$ pointwise agrees with some member of $U_{m_{1}}$ on $M$.

This is done by a standard diagonalization argument as in earlier proofs. We start with any moiety of the form $\bigcup_{n \in \mathbb{Z}}\left(a_{2 n}^{\prime}, a_{2 n+1}^{\prime}\right)$ where each $a_{n}^{\prime}$ lies in $C$, and express it as the disjoint union of an infinite sequence of moieties $\left(M_{i}: i \geq m_{0}\right)$. We shall show that $M$ in the statement of (4) may be taken as some $M_{i}$ with $i=m_{1} \geq m_{0}$. If not, then for each $i \geq m_{0}$ there is $g_{i} \in G_{C \backslash M_{i}}$ which does not agree with any member of $U_{i}$ on $M_{i}$. Let $g$ be the map on $\bigcup_{i \geq m_{0}} M_{i}$ obtained by 'patching' all these $g_{i} \mathrm{~s}$, that is, which agrees with $g_{i}$ on $M_{i}$ and which fixes all members of $C \backslash \bigcup_{n \in \mathbb{Z}}\left(a_{2 n}^{\prime}, a_{2 n+1}^{\prime}\right)$. By (2), $g$ extends to an automorphism of $\mathbb{D}$, also written as $g$. Since $G=\bigcup_{i \in \omega} U_{i}, g \in U_{i}$ for some $i \geq m_{0}$. But now $g$ and $g_{i}$ agree on $M_{i}$, which is a contradiction.

In what follows we fix this choice of $M$ and $\left(a_{n}\right)$.

(5) There is $m_{2} \geq m_{1}$ such that $G_{C \backslash M} \subseteq U_{m_{2}}$.

For this we choose $h \in G_{C \backslash M}$ which fixes each component of $M$ setwise but acts non-trivially there. Let $h \in U_{m}$. Since $\left[a_{2 n}, a_{2 n+1}\right]$ is isomorphic to the countable atomless boolean algebra, and also using (2) again, such $h$ exists. Now Anderson showed in [1] that the automorphism group of the countable atomless boolean algebra is simple, and furthermore, that for any non-identity elements $f$ and $f^{\prime}$, there are $f_{i}$ such that

$$
f^{\prime}=f^{f_{1}}\left(f^{-1}\right)^{f_{2}} f^{f_{3}}\left(f^{-1}\right)^{f_{4}} f^{f_{5}}\left(f^{-1}\right)^{f_{6}}
$$

(where superscripts indicate conjugation). Thus on each component of $M$, we may write any $g \in G_{C \backslash M}$ as a product of 6 conjugates of $h$ and its inverse of this form, and by (2), we may find $f_{i} \in G_{C \backslash M}$ such that $g=h^{f_{1}}\left(h^{-1}\right)^{f_{2}} h^{f_{3}}$ $\left(h^{-1}\right)^{f_{4}} h^{f_{5}}\left(h^{-1}\right)^{f_{6}}$. By (4), the $f_{i}$ agree with members $f_{i}^{\prime}$ of $U_{m_{1}}$ on $M$. Hence $g$ agrees with $h^{f_{1}^{\prime}}\left(h^{-1}\right)^{f_{2}^{\prime}} h^{f_{3}^{\prime}}\left(h^{-1}\right)^{f_{4}^{\prime}} h^{f_{5}^{\prime}}\left(h^{-1}\right)^{f_{6}^{\prime}}$ on $\mathbb{D}$. Since $h \in U_{m}$ and each $f_{i}^{\prime}$ lies in $U_{m_{1}}$, using $(\forall j, k)(\exists l) U_{j} U_{k} \subseteq U_{l}$, we find the desired $m_{2} \geq m, m_{1}$.

(6) There is $m_{3} \geq m_{2}$ such that for every moiety $M^{\prime}$ of the form $\bigcup_{n \in \mathbb{Z}}\left(a_{i_{2 n}}, a_{i_{2 n+1}}\right)$, where $i_{n}$ are integers such that $i_{n}<i_{n+1}$ for all $n$, $G_{C \backslash M^{\prime}} \subseteq U_{m_{3}}$.

To see the truth of this we let $m_{3}$ be such that $U_{m_{0}} U_{m_{2}} U_{m_{0}} \subseteq U_{m_{3}}$. Now we observe that there is $g \in G_{\{C\}}$ such that $g a_{n}=a_{i_{n}}$ for each $n$. Let $h \in G_{C \backslash M^{\prime}}$. Then $g^{-1} h g \in G_{C \backslash M}$, so by (5) lies in $U_{m_{2}}$. Hence $h \in g U_{m_{2}} g^{-1}$. But by (3), $g \in U_{m_{0}}$, so $h \in U_{m_{0}} U_{m_{2}} U_{m_{0}} \subseteq U_{m_{3}}$. 
(7) There is $m_{4} \geq m_{3}$ such that for every choice of $\left(i_{n}\right)$ as in (6), every automorphism fixing each $a_{i_{n}}$ lies in $U_{m_{4}}$.

Let $m_{4}$ be such that $U_{m_{3}} U_{m_{3}} \subseteq U_{m_{4}}$, and let $g \in G$ fix each $a_{i_{n}}$. Then $g$ may be written in the form $g_{2} g_{1}$ where $g_{1}$ fixes all members of $C \cap \bigcup_{n \in \mathbb{Z}}\left[a_{i_{2 n}}, a_{i_{2 n+1}}\right]$ and $g_{2}$ fixes all members of $C \cap \bigcup_{n \in \mathbb{Z}}\left[a_{i_{2 n+1}}, a_{i_{2 n+2}}\right]$. By (6), $g_{1}, g_{2} \in U_{m_{3}}$, and hence $g \in U_{m_{4}}$.

To conclude the proof, choose any $g \in G$. We can find a sequence of integers $\left(i_{n}\right)_{n \in \mathbb{Z}}$ such that for every $n$, both $a_{i_{n+1}}$ and $g\left(a_{i_{n+1}}\right)$ are greater than both $a_{i_{n}}$ and $g\left(a_{i_{n}}\right)$. Then for each $n, a_{i_{2 n+1}}$ and $g\left(a_{i_{2 n+1}}\right)$ lie between $a_{i_{2 n}}$ and $a_{i_{2 n+2}}$. Hence there is an automorphism $h$ fixing each $a_{i_{2 n}}$ and taking $g\left(a_{i_{2 n+1}}\right)$ to $a_{i_{2 n+1}}$. Now $h g$ fixes each $a_{i_{2 n+1}}$, so by $(7), g=h^{-1} h g \in$ $U_{m_{4}} U_{m_{4}} \subseteq U_{m_{5}}$, for some (fixed) $m_{5} \geq m_{4}$.

\section{The uncountable cofinality of the automorphism group of the countable universal homogeneous generalized boolean algebra}

We can use the same method to show the uncountable cofinality of the automorphism group of the closely related generalized boolean algebra. This is a combination of the proof given in the previous section, and the strong uncountable cofinality of the automorphism group of the countable atomless boolean algebra, established in [4] (remembering that this automorphism group is isomorphic to the group of homeomorphisms to itself of Cantor space).

Theorem 3.1. The automorphism group of the countable universal homogeneous generalized boolean algebra $\mathbb{B}$ has strong uncountable cofinality.

Proof. We first remark that in [6], some of the lemmas for $\mathbb{D}$ carry over to $\mathbb{B}$ with small modifications. For instance, Lemma 2.6 there says that three earlier results carry over with $\mathbb{Z}$-chains replaced by $\omega$-chains. The corresponding versions of (1) and (2) in the proof of Theorem 2.2 are thus as follows:

(1) For a suitable choice of maximal chain $C$ of $\mathbb{B}$, any isomorphism from $C$ to $C$ extends uniquely to an automorphism of $\mathbb{B}$.

As before, this $C$ may be chosen to be a maximal chain generating $\mathbb{B}$, and we let this be fixed for the rest of the proof.

(2) If $\left(a_{n}\right),\left(a_{n}^{\prime}\right)$ are cofinal $\omega$-sequences in $C$ such that $a_{0}=a_{0}^{\prime}=0$, then any isomorphism from $C \cup \bigcup_{n \in \omega}\left[a_{2 n}, a_{2 n+1}\right]$ to $C \cup \bigcup_{n \in \omega}\left[a_{2 n}^{\prime}, a_{2 n+1}^{\prime}\right]$ (or from $C \cup \bigcup_{n \in \omega}\left[a_{2 n+1}, a_{2 n+2}\right]$ to $\left.C \cup \bigcup_{n \in \omega}\left[a_{2 n+1}^{\prime}, a_{2 n+2}^{\prime}\right]\right)$ extends uniquely to an automorphism of $\mathbb{B}$. 
We continue with versions of the other steps in the proof of Theorem 2.2. The proofs of (3), (4), (5), (6), and (7) are as before.

(3) There is $m_{0}$ such that $G_{\{C\}} \subseteq U_{m_{0}}$.

(4) There are a moiety $M=\bigcup_{n \in \omega}\left(a_{2 n+1}, a_{2 n+2}\right)$ such that each $a_{n}$ lies in $C$, and $m_{1} \geq m_{0}$, such that every automorphism fixing $C \backslash M$ pointwise agrees with some member of $U_{m_{1}}$ on $M$.

This time, by 'moiety' we mean a subset of $\mathbb{B}$ of the form $\bigcup_{n \in \omega}\left(a_{2 n+1}, a_{2 n+2}\right)$ where $\left(a_{n}\right)$ is a cofinal $\omega$-sequence.

From now on, $M$ and the sequence $\left(a_{n}\right)$ are fixed.

(5) There is $m_{2} \geq m_{1}$ such that $G_{C \backslash M} \subseteq U_{m_{2}}$.

We also need a version of this for 'moieties' which may include the bottom level.

$\left(5^{\prime}\right)$ There is $m_{3} \geq m_{2}$ such that $G_{C \backslash M^{\prime}} \subseteq U_{m_{3}}$, where $M^{\prime}=$ $\bigcup_{n \in \omega}\left(a_{2 n}, a_{2 n+1}\right)$.

Since $\left[a_{0}, a_{1}\right]$ is isomorphic to the countable atomless boolean algebra, by [4], Aut $\left(\left[a_{0}, a_{1}\right]\right)$ has strong uncountable cofinality. By considering the intersections of the sequence $\left(U_{n}\right)$ with $G_{C \backslash\left[a_{0}, a_{1}\right]}$ (which by (2) is isomorphic to $\left.\operatorname{Aut}\left(\left[a_{0}, a_{1}\right]\right)\right)$ we find $m^{\prime}$ such that $G_{C \backslash\left[a_{0}, a_{1}\right]} \subseteq U_{m^{\prime}}$. Choose $m_{3} \geq m_{2}$ such that $U_{m_{0}} U_{m_{2}} U_{m_{0}} U_{m^{\prime}} \subseteq U_{m_{3}}$.

Now let $g \in G_{C \backslash M^{\prime}}$. Then we may write $g$ as $g_{2} g_{1}$ where $g_{1}$ fixes all members of $C \backslash\left[a_{0}, a_{1}\right]$ and $g_{2}$ fixes all members of $C \backslash \bigcup_{n \geq 1}\left[a_{2 n}, a_{2 n+1}\right]$. Let $h \in G_{\{C\}}$ map $a_{n}$ to $a_{n+1}$ for all $n \geq 1$. Then $h^{-1} g_{2} h$ fixes all members of $C \backslash M$, so by (5), lies in $U_{m_{2}}$. Hence $g=h\left(h^{-1} g_{2} h\right) h^{-1} g_{1} \in U_{m_{0}} U_{m_{2}} U_{m_{0}} U_{m^{\prime}}$ $\subseteq U_{m_{3}}$.

(6) There is $m_{4} \geq m_{3}$ such that for every moiety $M^{\prime}$ of the form $\bigcup_{n \in \omega}\left(a_{i_{2 n+1}}, a_{i_{2 n+2}}\right)$, where $i_{n}$ are natural numbers such that $i_{n}<i_{n+1}$ for all $n, G_{C \backslash M^{\prime}} \subseteq U_{m_{3}}$.

(7) There is $m_{5} \geq m_{4}$ such that for every choice of $\left(i_{n}\right)$ as in (6), every automorphism fixing each $a_{i_{n}}$ lies in $U_{m_{5}}$.

The proof is concluded as in Theorem 2.2.

We remark in conclusion that in [7], we established the strong uncountable cofinality for various uncountable chains. It is tempting to ask whether these results extend to the distributive lattices generated by these chains, in the style of the present paper. It would be necessary to derive results about simplicity similar to those in [1], in a strong version providing explicitly for expressions using finitely many conjugates, such as we used in step (5) of the proof of Theorem 2.2. 


\section{References}

[1] R. D. Anderson, The algebraic simplicity of certain groups of homeomorphisms, Amer. J. Math. 80 (1958), 955-963.

[2] G. M. Bergman, Generating infinite symmetric groups, Bull. London Math. Soc. 38 (2006), 429-440.

[3] J. D. Dixon, P. M. Neumann, S. Thomas, Subgroups of small index in infinite permutation groups, Bull. London Math. Soc. 18 (1986), 580-586.

[4] M. Droste, R. Göbel, Uncountable cofinalities of permutation groups, J. London Math. Soc. 71 (2005), 335-344.

[5] M. Droste, W. C. Holland, Generating automorphism groups of chains, Forum Math. 17 (2005), 699-710.

[6] M. Droste, D. Macpherson, The automorphism group of the universal distributive lattice, Algebra Universalis 43 (2000), 295-306.

[7] M. Droste, J. K. Truss, Uncountable cofinalities of automorphism groups of linear and partial orders, Algebra Universalis 62 (2009), 75-90.

[8] A. M. W. Glass, Ordered Permutation Groups, London Math. Soc. Lecture Notes No. 55 (1981), Cambridge University Press.

[9] G. A. Grätzer, General Lattice Theory, 2nd edition, Birkhäuser Verlag, Basel, 2003.

[10] C. Gourion, À propos du groupe des automorphismes de $(\mathbb{Q}, \leq)$, C. R. Acad. Sci. Paris 315 (1992), 1329-1331.

[11] W. Hodges, Model Theory, Cambridge University Press, Cambridge, 1993.

[12] A. S. Kechris, C. Rosendal, Turbulence, amalgamation, and generic automorphisms of homogeneous structures, Proc. London Math. Soc. 94 (2006), 302-350.

[13] S. Koppelberg, J. Tits, Une propriété des produits directs infinis de groupes finis isomorphes, C. R. Acad. Sci. Paris 279 (1974), 583-585.

[14] H. D. Macpherson, P. M. Neumann, Subgroups of infinite permutation groups, J. London Math. Soc. 42 (1990), 64-84.

[15] S. Thomas, Cofinalities of infinite permutation groups, in: Advances in Algebra and Model Theory, edited by M. Droste and R. Göbel, Gordon and Breach, pages 101-120, 1997.

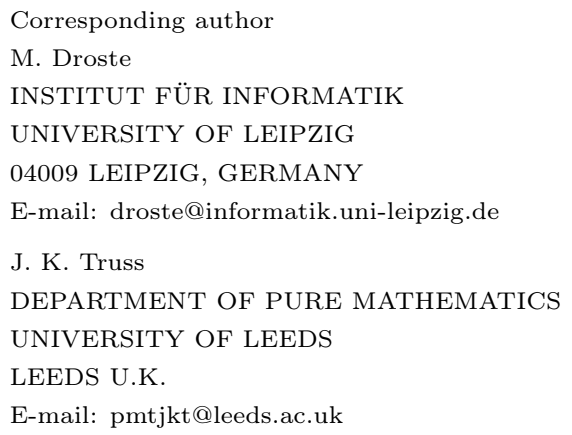

Received July 6, 2010; revised version July 27, 2010. 